\title{
A Retrospective Analysis of Real-World Discontinuation Rates with Delayed-Release Dimethyl Fumarate in Patients with Relapsing-Remitting Multiple Sclerosis
}

\author{
Michelle Allan · Lindsay Grant
}

Received: October 14, 2019 / Published online: December 13, 2019

(C) The Author(s) 2019

\section{ABSTRACT}

Introduction: The main objective of this study was to examine discontinuation rates associated with delayed-release dimethyl fumarate (DMF) when used for the treatment of relapsing multiple sclerosis (MS) in a real-world, clinical practice setting.

Methods: Data were collected retrospectively from charts of adult patients with relapsing-remitting MS treated at a single large institution in Australia, who completed at least 6 months of continuous therapy, either with DMF or another MS medication administered following DMF discontinuation. The primary endpoint was overall discontinuation rate. Secondary endpoints included discontinuation rate 6 months after initiation of DMF therapy;

Enhanced Digital Features To view enhanced digital features for this article go to https://doi.org/10.6084/ m9.figshare.11206007.

M. Allan ( $\square)$

Monash Health, Clayton, VIC 3168, Australia

e-mail: michelle.allan@monashhealth.org

L. Grant

Biogen, North Ryde, NSW 2066, Australia incidence of adverse events, particularly gastrointestinal events; discontinuation rate because of adverse events; and use of concomitant medications by patients during administration of DMF.

Results: A total of 100 patients initially prescribed DMF between October 1, 2013 and June 30,2014 were included in the analysis. The mean age of the patients was 43 years and $80 \%$ were female. The overall discontinuation rate was $13 \%$, with $9 \%$ discontinuing because of gastrointestinal tolerability issues, within the first 6 months. Dose changes as a result of adverse events occurred in $15 \%$ of patients, and none of the adverse events reported were serious. Only one patient discontinued owing to lack of efficacy.

Conclusion: This study, conducted shortly after the approval of DMF in Australia when firsthand clinical experience was still limited, demonstrated that DMF has an acceptable tolerability profile in the real-world setting that is similar to that demonstrated in clinical trials.

Keywords: Adverse events; Delayed-release dimethyl fumarate; Discontinuation; Diseasemodifying agents; Multiple sclerosis; Real world 


\section{Key Summary Points}

Why carry out this study?

Delayed-release dimethyl fumarate (DMF) is an oral disease-modifying therapy (DMT) for the treatment of multiple sclerosis (MS)

DMF has demonstrated efficacy and safety in clinical trials and real-world studies, but gastrointestinal intolerance and flushing are commonly reported in the first few months of treatment and may lead to DMF discontinuation

We conducted a retrospective chart review to obtain a better understanding of the incidence and impact of these adverse events in the realworld setting

\section{What was learned from the study?}

In a total of 100 patients initially prescribed DMF, the overall discontinuation rate was $13 \%$ within 6 months of initiation, with $9 \%$ discontinuing because of gastrointestinal tolerability issues

This study, conducted shortly after the approval of DMF in Australia when firsthand clinical experience was still limited, demonstrated that DMF has an acceptable tolerability profile in the realworld setting that is similar to that demonstrated in clinical trials

\section{INTRODUCTION}

Delayed-release dimethyl fumarate (DMF; also known as gastro-resistant DMF) is an oral disease-modifying therapy (DMT) for the treatment of multiple sclerosis (MS) that is administered at a dose of $240 \mathrm{mg}$ twice daily. The efficacy of DMF has been demonstrated in two phase 3 clinical trials, which showed that
DMF reduced relapse rates and improved neuroradiological outcomes, such as lesions, compared with placebo $[1,2]$. The most commonly reported adverse events in clinical trials were flushing and gastrointestinal (GI) intolerance $[1,2]$. Furthermore, GI intolerance and flushing were the most common reasons for discontinuation, accounting for $4 \%$ and $3 \%$ of discontinuations, respectively [3]. The incidence of GI events (mainly diarrhea, nausea, and abdominal pain) and flushing was greater early in the course of treatment (primarily in the first month) and decreased over time [3, 4]. The GI tolerability of DMF was further explored in the multicenter, open-label, single-arm MANAGE study, which demonstrated that $7.3 \%$ of patients discontinued treatment because of GIrelated adverse events [5]. It is hypothesized, and as yet not tested clinically, that the chemical structure of DMF could potentially elicit irritation in the GI tract through production of methanol, a GI-irritating pro-moiety, and reactivity toward off-target receptors [6].

We conducted a retrospective chart review to obtain a better understanding of the incidence and impact of these adverse events in the realworld setting. The main objective was to examine discontinuation rates for patients with relapsing-remitting MS, who were initiated on DMF and had received at least 6 months of treatment for their condition. This study was conducted shortly after the approval of DMF in Australia when first-hand clinical experience with this treatment was still limited.

\section{METHODS}

\section{Study Design}

The study was designed as a clinical care initiative in the form of a chart review of patients with relapsing-remitting MS who received care at a single large medical institution in Australia, the Monash MS Clinic. Data were collected retrospectively by institution staff and in a deidentified manner from patients' medical charts and related information sources such as physicians' notes and pharmacy records. All data 
collected for the clinical care initiative were then entered onto a paper case report form.

Data collected included patient demographic information, including age, gender, and ethnicity; patient history of MS (including date of diagnosis of relapsing-remitting MS) and age at the time of diagnosis; other comorbidities of interest, including osteoporosis, depression, and anxiety; DMTs used prior to initiation of DMF; date DMF was first prescribed, changes in dosage, and use of concomitant medications during the period of DMF administration; and a date DMF was discontinued, reason for discontinuation (if recorded), the DMT replacing DMF, and concomitant medication use during the period following DMF discontinuation.

\section{Patients}

Charts were reviewed to determine patients who met the following criteria: (1) diagnosed with relapsing-remitting MS; (2) initially prescribed DMF therapy between October 1, 2013 and June 30, 2014; (3) completed at least 6 months of continuous therapy for their MS, either with DMF or an alternative MS medication initiated following discontinuation of DMF therapy; (4) experienced no change in their MS diagnosis during the 6-month follow-up period; and (5) at least 18 years of age at the start of DMF therapy.

\section{Study Endpoints}

The primary endpoint was overall discontinuation rate for patients with MS treated with DMF. Given the retrospective design of the study, "discontinuation" was defined as information in the patient's chart (or related patient information sources such as physicians' notes or pharmacy records) indicating either that the patient stopped receiving DMF for at least 1 week or that DMF was terminated.

Secondary endpoints included discontinuation rate 6 months after initiation of DMF therapy; incidence of adverse events, particularly GI events; discontinuation rate due to adverse events; and use of concomitant medications by patients during administration of DMF.

\section{Statistical Analysis}

Descriptive statistics were used to report information about the overall discontinuation rate at 3 and 6 months following initiation of DMF. Within the limits of data collected retrospectively from patient charts and the potential for missing information, efforts were made to assess the basis for DMF discontinuation and the factors associated with discontinuation, such as previous therapy and MS severity.

Adverse events, serious adverse events, and concomitant medications of interest were also recorded and tabulated, including the number of events and the respective number of patients who experienced an event at least once. To the degree possible, adverse events were categorized on the basis of their relationship to DMF as follows: definitely related, probably related, not related, and unable to determine.

Table 1 Patient demographics ${ }^{a}$

\begin{tabular}{ll}
\hline Characteristics & Parameters \\
\hline Age, years & $43(11)$ \\
Mean (SD) & $44(18-65)$ \\
Median (range) & 80 \\
Female, \% & \\
Disease duration, years & $8.5(7)$ \\
Mean (SD) & $7(0-29)$ \\
Median (range) & 16 \\
Number of prior DMTs, \% & 45 \\
0 & 26 \\
1 & 8 \\
2 & 3 \\
3 & 2 \\
\hline & \\
\hline &
\end{tabular}


Table 2 Reason for discontinuing prior disease-modifying therapy

\begin{tabular}{|c|c|c|c|c|c|c|c|}
\hline Agent, $n(\%)$ & $\begin{array}{l}\text { Adverse } \\
\text { event }\end{array}$ & $\begin{array}{l}\text { Lack of } \\
\text { efficacy }\end{array}$ & $\begin{array}{l}\text { Injection } \\
\text { fatigue }\end{array}$ & $\begin{array}{l}\text { Request to go to } \\
\text { oral treatment }\end{array}$ & $\begin{array}{l}\text { Poor } \\
\text { tolerability }\end{array}$ & Pregnancy & Other \\
\hline Teriflunomide $(n=9)$ & $2(22.2)$ & $2(22.2)$ & $1(11.1)$ & - & $3(33.3)$ & $1(11.1)$ & 0 \\
\hline $\begin{array}{l}\text { Interferon beta-1a IM } \\
\text { QW }(n=30)\end{array}$ & $3(10.0)$ & $11(36.7)$ & $3(10.0)$ & $4(13.3)$ & $6(20.0)$ & 0 & $3(10.0)$ \\
\hline $\begin{array}{l}\text { Interferon beta- } 1 \mathrm{~b} \\
\quad(n=29)\end{array}$ & $4(13.8)$ & $10(34.5)$ & $7(24.1)$ & $2(6.9)$ & $3(10.3)$ & $1(3.4)$ & $1(3.4)$ \\
\hline $\begin{array}{l}\text { Glatiramer acetate } \\
\quad(n=30)\end{array}$ & $7(23.3)$ & $13(43.3)$ & $3(10.0)$ & $2(6.7)$ & $3(10.0)$ & 0 & $1(3.3)$ \\
\hline Fingolimod $(n=17)$ & $6(35.3)$ & $7(41.2)$ & 0 & - & $2(11.8)$ & $1(5.9)$ & $1(5.9)$ \\
\hline $\begin{array}{l}\text { Interferon beta-1a SC } \\
\text { TIW }(n=19)\end{array}$ & $3(15.8)$ & $6(31.6)$ & $2(10.5)$ & $3(15.8)$ & $3(15.8)$ & 0 & 0 \\
\hline Natalizumab $(n=9)$ & 0 & $1(11.1)$ & 0 & 0 & $1(11.1)$ & 0 & $7(77.8)$ \\
\hline $\begin{array}{l}\text { Total }(N=143)^{\mathrm{a}}, \\
n(\%)\end{array}$ & $25(17)$ & $50(35)$ & $16(11)$ & $11(8)$ & $21(15)$ & $3(2)$ & $13(9)$ \\
\hline
\end{tabular}

Data are $n(\%)$ unless otherwise specified

$I M$ intramuscular, $Q W$ once weekly, $S C$ subcutaneous, $T I W$ three times weekly

a $N=100$ patients; patients could have discontinued from $>1$ prior disease-modifying therapy and could have given $>1$ reason for discontinuing a prior therapy

\section{Ethics Approval}

The study was approved as a low risk study by the Research Support Services, Monash Health, and was conducted in accordance with the ethical principles of the Declaration of Helsinki. Informed consent was not required because this is a retrospective chart review.

\section{RESULTS}

\section{Demographic Characteristics}

The analysis included 100 charts of consecutive patients initially prescribed DMF between October 1, 2013 and June 30, 2014. Demographic characteristics are summarized in Table 1. Overall, most (84\%) patients had received one or more prior DMTs when initiating DMF. The majority (67\%) of prior DMTs had been used for more than 12 months. The most common reasons for discontinuing prior DMTs were lack of efficacy (50\%), adverse events (25\%), and poor tolerability (21\%; Table 2$)$.

\section{Discontinuation Rates}

The discontinuation rate was $13 \%$ at 6 months after DMF initiation, mostly because of adverse events (Fig. 1); none of the adverse events reported were serious. In total, 9\% of patients discontinued because of GI tolerability issues. Only one patient discontinued DMF because of lack of efficacy. Most patients used one or more concomitant medications, the most common being aspirin; the next most commonly used was for abdominal pain (used in 5\% of patients; Table 3).

No DMT-naive patients discontinued therapy with DMF within 6 months of initiation. Five of the 13 patients who discontinued DMF within 6 months of therapy initiation had received one prior DMT, compared with eight who had received two or three prior DMTs. Two patients did not start another DMT within the follow-up time period of the data collection. Of patients 


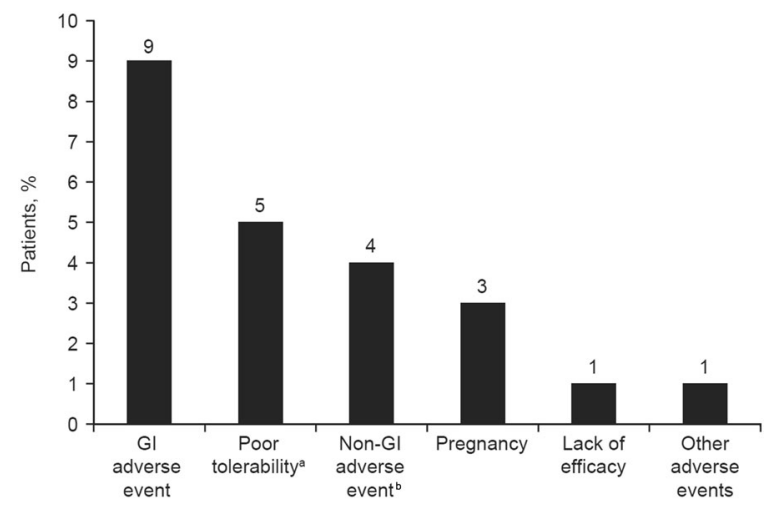

Fig. 1 Reasons for discontinuation of delayed-release dimethyl fumarate $(N=13)$. GI gastrointestinal. ${ }^{\text {a }}$ All five patients also discontinued because of adverse events. ${ }^{\mathrm{b}}$ Flushing is included as a non-GI adverse event

Table 3 Use of concomitant medications during treatment with disease-modifying therapy

\begin{tabular}{ll}
\hline Medication & Patients, $\boldsymbol{n}$ \\
\hline Aspirin & 84 \\
Medications for abdominal pain & 5 \\
Dopamine agonists & 4 \\
Antihistamines & 1 \\
Antacids & 3 \\
Antidiarrheal medications & 3 \\
Proton pump inhibitors & 2 \\
Serotonin 5-HT ${ }^{3}$ antagonists & 2 \\
\hline
\end{tabular}

with a history of discontinuing prior DMT because of poor tolerability, the majority $(82 \%)$ remained on DMF therapy at 6 months. A total of 15 patients had their dose changed because of an adverse event; four of these patients discontinued DMF in the first 6 months of therapy. Among patients who discontinued DMF, three patients switched to teriflunomide, three to fingolimod, two to natalizumab, one to alemtuzumab, and two to glatiramer acetate for injection.

\section{DISCUSSION}

In this retrospective chart review reflecting realworld use of DMF in a single large institution in
Australia, $13 \%$ of patients discontinued therapy within 6 months of initiation. In real-world analyses, discontinuation rates at 1 year ranged from $10 \%$ to $29 \%$ for DMF [5, 7-12] and $11 \%$ to $24 \%$ for other DMTs, including glatiramer acetate, fingolimod, interferons, natalizumab, and teriflunomide [8, 10-13]. Although this analysis assessed discontinuation rate at 6 months, the majority (81\%) of discontinuations for DMF occurred within the first 5 weeks, suggesting that the discontinuation rate would not rise substantially from 6 to 12 months [14]. The most common reason for discontinuation was GI events (9\%), as was seen in two previous phase 3 clinical trials assessing this agent [3]. The discontinuation rate due to GI events in these trials was $4.3 \%$. In the multicenter, openlabel, single-arm MANAGE study designed to further explore the tolerability of DMF by evaluating GI-related events, a total of $9.9 \%$ of patients discontinued as a result of adverse events, with 7.3\% discontinuing because of GIrelated adverse events [5].

The most commonly reported GI events in the phase 3 clinical trials were diarrhea, nausea, and abdominal pain [3]. In the MANAGE study, the most common GI-related events leading to discontinuation were nausea, diarrhea, and vomiting [5]. Although specific adverse events were not reported in the current study, it can be hypothesized by examining the use of concomitant medications that the most common GI event was abdominal pain followed by nausea and vomiting, because the most commonly prescribed medications were for GI pain and discomfort.

The Monash MS Clinic has a dedicated MS nurse who provides patient support, including telephone support, to all newly diagnosed patients and any patients starting, switching, or continuing therapy. When initiating DMF, it is also recommended that patients enroll in a patient support program so that they can receive additional support. Support program nurses and the Monash MS Clinic nursing service communicate regularly regarding the support and education of patients treated with DMF.

The main goal of supporting and educating patients during the first month of therapy with 
Table 4 Consensus delayed-release dimethyl fumarate titration protocol

\begin{tabular}{ll}
\hline Week & Dose \\
\hline Week 1 & $120 \mathrm{mg}$ QD \\
Week 2 & $240 \mathrm{mg}$ QD \\
Week 3 & $120 \mathrm{mg}$ morning; 240 mg night \\
Week 4 & $240 \mathrm{mg}$ BID \\
\hline
\end{tabular}

$B I D$ twice daily, $Q D$ once daily

DMF is to ensure that any tolerability issues are quickly identified and addressed. Education and the setting of realistic expectations have been shown to be critical to maintaining treatment persistence $[3,15]$. Patients are educated to contact the support program or the Monash MS Clinic at the first sign of any GI side effects and/ or flushing so that these can be managed using a variety of interventions. These may include taking aspirin $30 \mathrm{~min}$ before DMF to reduce flushing; having appropriate food in their stomach before taking DMF; administration of hyoscine butylbromide to reduce GI spasm; and slower retitration after a small break in treatment $[3,5,16,17]$. Furthermore, it has been demonstrated that counselling and setting the patient's expectations up front is critical in managing GI side effects $[5,18]$.

When DMF first became available at the Monash MS Clinic, patients started treatment as per the instructions in the product information [3]. Anecdotally, about a third of patients experienced a GI adverse event that was serious enough to report to nursing staff (either a support program or Monash MS Clinic nurse). Consultation with other centers led to the adoption of a titration protocol (Table 4), which involved increasing the dose of DMF more slowly than recommended in the product information. This titration regimen has provided time and cost savings by reducing the need for the physicians to utilize the $120 \mathrm{mg}$ starter dose, as well as a reduction in the nurse's time to manage adverse events. A US study utilizing a similar, nurse-designed protocol demonstrated a significantly reduced number of discontinuations from 14 (12\%) with the standard manufacturer-recommended protocol to five $(2.5 \%)$ in patients using the slower titration protocol $(p=0.0029)$. Discontinuations related to GI side effects were also significantly reduced from $10(8 \%)$ to four $(1.9 \%)$, respectively ( $p=0.0215)$ [19].

There are some limitations to this real-world study, including that the population consisted entirely of patients treated at one large institution. Furthermore, data collected retrospectively from patient charts always has the potential for missing information (e.g., specific adverse events that were not reported). In addition, the study was conducted when DMF was first introduced to Australia, and since then many centers have adopted different practices to reduce the risk of GI side effects.

\section{CONCLUSIONS}

The current study demonstrated that DMF for the treatment of relapsing-remitting MS has acceptable tolerability in a real-world, clinical practice setting. Although this study was conducted shortly after the approval of DMF in Australia when first-hand clinical experience was still limited, the discontinuation rate was comparable to that seen in two phase 3 clinical trials and an open-label study. This included discontinuations because of GI-related adverse events. The majority of patients with a history of poor tolerability to prior therapy did not discontinue therapy with DMF within 6 months of initiation, suggesting that history of prior intolerability issues is not an indicator of DMF tolerability. The adoption of strategies initiated by the Monash MS Clinic to minimize the risk of discontinuation, including nurse-led patient education and support and a dose titration protocol, likely played a key role in maintaining patients on DMF therapy.

\section{ACKNOWLEDGEMENTS}

The authors wish to thank the patients for the use of their data in this study. 
Data Availability. The datasets generated during and/or analyzed during the current study are available from the corresponding author on reasonable request.

Funding. This study and the journal's Rapid Service were supported by Biogen (New South Wales, Australia).

Medical Writing Assistance. Anne Burridge, a freelance medical writer, wrote the first draft of the manuscript based on input from the authors. Biogen provided funding for medical writing support in the development of this manuscript. Biogen reviewed and provided feedback on the manuscript.

Authorship. All named authors meet the International Committee of Medical Journal Editors (ICMJE) criteria for authorship for this article, take responsibility for the integrity of the work as a whole, and have given their approval for this version to be published.

Disclosures. Michelle Allan has nothing to disclose. Lindsay Grant is a full-time employee of and holds stock/stock options in Biogen.

Compliance with Ethics Guidelines. The study was approved as a low risk study by the Research Support Services, Monash Health, and was conducted in accordance with the ethical principles of the Declaration of Helsinki. Informed consent was not required because this is a retrospective chart review.

Open Access. This article is distributed under the terms of the Creative Commons Attribution-NonCommercial 4.0 International License (http://creativecommons.org/licenses/ by-nc/4.0/), which permits any noncommercial use, distribution, and reproduction in any medium, provided you give appropriate credit to the original author(s) and the source, provide a link to the Creative Commons license, and indicate if changes were made.

\section{REFERENCES}

1. Fox RJ, Miller DH, Phillips JT, et al. Placebo-controlled phase 3 study of oral BG-12 or glatiramer in multiple sclerosis. N Engl J Med. 2012;367(12): 1087-97. https://doi.org/10.1056/NEJMoa1206328.

2. Gold R, Kappos L, Arnold DL, et al. Placebo-controlled phase 3 study of oral BG-12 for relapsing multiple sclerosis. N Engl J Med. 2012;367(12): 1098-107.

NEJMoa1114287.

3. Biogen Australia Pty Ltd. Tecfidera Australian product information. http://www.guildlink.com.au/ gc/ws/bd/rss.cfm?product=bdptecfi10917. Accessed Feb 62017.

4. Phillips JT, Selmaj K, Gold R, et al. Clinical significance of gastrointestinal and flushing events in patients with multiple sclerosis treated with delayed-release dimethyl fumarate. Int J MS Care. 2015;17(5):236-43. https://doi.org/10.7224/15372073.2014-069.

5. Fox EJ, Vasquez A, Grainger W, et al. Gastrointestinal tolerability of delayed-release dimethyl fumarate in a multicenter, open-label study of patients with relapsing forms of multiple sclerosis (MANAGE). Int J MS Care. 2016;18(1):9-18. https:// doi.org/10.7224/1537-2073.2014-101.

6. Palte MJ, Wehr A, Tawa $\mathrm{M}$, et al. Improving the gastrointestinal tolerability of fumaric acid esters: early findings on gastrointestinal events with diroximel fumarate in patients with relapsingremitting multiple sclerosis from the phase 3, openlabel EVOLVE-MS-1 study. Adv Ther. 2019;36(11): 3154-65. https://doi.org/10.1007/s12325-01901085-3.

7. Min J, Cohan S, Alvarez E, et al. Real-world characterization of dimethyl fumarate-related gastrointestinal events in multiple sclerosis: management strategies to improve persistence on treatment and patient outcomes. Neurol Ther. 2019. https://doi. org/10.1007/s40120-019-0127-2.

8. Braune $S$, Grimm S, van Hovell $P$, et al. Comparative effectiveness of delayed-release dimethyl fumarate versus interferon, glatiramer acetate, teriflunomide, or fingolimod: results from the German NeuroTransData registry. J Neurol. 2018;265(12): 2980-92. https://doi.org/10.1007/s00415-0189083-5.

9. Kresa-Reahl K, Repovic P, Robertson D, Okwuokenye M, Meltzer L, Mendoza JP. Effectiveness of delayed-release dimethyl fumarate on clinical and patient-reported outcomes in patients with relapsing multiple sclerosis switching from glatiramer 
acetate: RESPOND, a prospective observational study. Clin Ther. 2018;40(12):2077-87. https://doi. org/10.1016/j.clinthera.2018.10.011.

10. Vu NBM, Moses H, Sriram S, Pawate S. Efficacy and tolerability of fingolimod, dimethyl fumarate, and teriflunomide in patients with multiple sclerosis: real world experience from a single center. Neurology. 2016;86(Suppl 16):P6.168.

11. Ontaneda DVB, Sillau S, et al. Comparative efficacy and discontinuation of fingolimod and dimethyl fumarate in two large academic medical centers. Neurology. 2016;86(Suppl 16):P3.109.

12. Vollmer B, Nair KV, Sillau SH, Corboy J, Vollmer T, Alvarez E. Comparison of fingolimod and dimethyl fumarate in the treatment of multiple sclerosis: two-year experience. Mult Scler J Exp Transl Clin. 2017;3(3):2055217317725102. https://doi.org/10. $1177 / 2055217317725102$.

13. Frisell T, Forsberg L, Nordin N, et al. Comparative analysis of first-year fingolimod and natalizumab drug discontinuation among Swedish patients with multiple sclerosis. Mult Scler. 2016;22(1):85-93. https://doi.org/10.1177/1352458515579216.

14. Min J, Cohan S, Alvarez E, et al. Real-world characterization of dimethyl fumarate-related gastrointestinal events in multiple sclerosis: management strategies to improve persistence on treatment and patient outcomes. Neurol Ther. 2019;8(1):109-19. https://doi.org/10.1007/s40120-019-0127-2.

15. Begus-Nahrman Y, Niemczyk G, Schmid B, Mäurer $M$. The potential of individualized patient coaching to optimize treatment with delayed-release dimethyl fumarate: a retrospective analysis of patients with multiple sclerosis treated in a realworld setting. Poster presented at 32nd Congress of the European Committee for Treatment and Research in Multiple Sclerosis (ECTRIMS) 2016 Meeting; September 14-17, 2016; London, UK.

16. O'Gorman J, Russell HK, Li J, Phillips G, Kurukulasuriya NC, Viglietta V. Effect of aspirin pretreatment or slow dose titration on flushing and gastrointestinal events in healthy volunteers receiving delayed-release dimethyl fumarate. Clin Ther. 2015;37(7):1402-19.e5. https://doi.org/10. 1016/j.clinthera.2015.03.028.

17. Theodore Phillips J, Erwin AA, Agrella S, et al. Consensus management of gastrointestinal events associated with delayed-release dimethyl fumarate: a Delphi study. Neurol Ther. 2015;4(2):137-46. https://doi.org/10.1007/s40120-015-0037-X.

18. Min J, Sloane J, Okwuokenye M, Taylor C, Theodore Phillips J. Real-world strategies for managing gastrointestinal events and increasing adherence with delayed-release dimethyl fumarate: interim analysis of a substudy of EFFECT. Poster presented at Consortium of Multiple Sclerosis Centers 2017 Annual Meeting; May 24-27, 2017; New Orleans, LA, USA.

19. Sammarco C, Laing L, Minetti J, Desanctis C, Herbert J. Strategies to reduce adverse events related to oral dimethyl fumarate. Poster presented at Americas Committee for Treatment and Research in Multiple Sclerosis (ACTRIMS)-European Committee for Treatment and Research in Multiple Sclerosis (ECTRIMS) 2014 Meeting; September 10-13, 2014; Boston, MA, USA. 